\title{
Oscillatory Size-Dependence of the Surface Plasmon Linewidth in Metallic Nanoparticles
}

\author{
Rafael A. Molina, ${ }^{1,2}$ Dietmar Weinmann, ${ }^{2}$ and Rodolfo A. Jalabert ${ }^{2}$ \\ ${ }^{1}$ Departamento de Física Atómica, Molecular y Nuclear, \\ Universidad Complutense de Madrid, 28040 Madrid, Spain \\ ${ }^{2}$ Institut de Physique et Chimie des Matériaux de Strasbourg, \\ UMR 7504 (CNRS-ULP), 23 rue du Loess, 67037 Strasbourg, France
}

\begin{abstract}
We study the linewidth of the surface plasmon resonance in the optical absorption spectrum of metallic nanoparticles, when the decay into electron-hole pairs is the dominant channel. Within a semiclassical approach, we find that the electron-hole density-density correlation oscillates as a function of the size of the particles, leading to oscillations of the linewidth. This result is confirmed numerically for alkali and noble metal particles. While the linewidth can increase strongly, the oscillations persist when the particles are embedded in a matrix.

PACS numbers: PACS: 71.45.Gm, 36.40.Vz, 31.15.Gy
\end{abstract}

\section{INTRODUCTION}

The lifetime of collective excitations in finite quantum systems is a fundamental problem spanning from the physics of nuclei to that of metallic clusters. Giant resonances, in the former casel, and surface plasmon, in the latter $\boldsymbol{B}$, decay by different mechanisms according to the physical regime of the excitation. For large metallic clusters (with radius $R \gtrsim 10 \mathrm{~nm}$ ) radiation damping is the main limiting factor to the plasmon lifetimed. At small sizes $(0.5 \mathrm{~nm} \lesssim R \lesssim 2 \mathrm{~nm})$ the decay into electron-hole pairs (Landau damping) dominates, while for intermediate sizes both effects compete. For very small clusters $(R \lesssim 0.5 \mathrm{~nm})$ a strong temperature dependence of the linewidth 5 suggests that the coupling of the collective excitation to thermal vibrations of the atomic lattice is an important effect.

Except for the case of very small clusters, where the atomic arrangements play a crucial role, the theoretical analysis of the surface plasmon is usually based on the jellium model, where conduction electrons move in a uniformly charged background. Within such a framework, the Landau damping occurs when there are particle-hole excitations with an energy close to that of the collective excitation. Therefore, this mechanism is strongly dependent on the density of states of the conduction electrons confined in the cluster.

Using a linear response approach, Kawabata and Kubde proposed a total linewidth $\Gamma=\Gamma_{\mathrm{i}}+\Delta \Gamma(R)$, where the intrinsic width $\Gamma_{\mathrm{i}}$ is given by bulk properties and the size-dependent contribution $\Delta \Gamma(R)$ is proportional to $1 / R$. Such an $R$ dependence would also result from the identification of the diameter of the particle with the electron mean-free-path. However, as stressed in the original work, this identification is not completely correct, since the quantized states exist due to the confinement and cannot be considered to be scattered at the surface 16. On the other hand, for interacting electrons confined in a parabolic potential, Kohn's theorem implies an infinitely-lived collective excitations, such that the linewidth of the plasmon can be thought as a measure of the failure in separating the collective (center-of-mass) and relative coordinates for the electron system.

Further improvements in the linear response theory by Yannouleas and Brogliad and by Barma and Subrahmanyam 10 recovered the $1 / R$ dependence of $\Delta \Gamma(R)$ in the asymptotic limit of large $R$ Numerical calculation 10.11 and existing experiments 12 on free intermediate-size clusters are in good agreement with the values of $\Delta \Gamma(R)$ yielded by those calculations. For small clusters the emergence of a non-monotonous size-dependent structure in the lineshape (in exmeriment, 13. 4.45 as well as in numerical calculations $10.11,16,17.18$ ) has heen attributed to shell effects (see also Fig. 10 of Ref.11 a). We provide in this work a detailed theoretical understanding of these effects and determine analytically the sizedependence of the plasmon lifetime for free and embedded clusters.

Using a semiclassical approach, we explain how a subtle consequence of electronic shell effects, namely angular-momentum dependent electron-hole density-density correlations, leads to an oscillatory size-dependence of the plasmon linewidth due to Landau damping in small alkaline metal clusters. This result is confirmed by numerical calculations using the time-dependent local density approximation (TDLDA) 11 19. We show that our results are relevant also for noble metals and clusters embedded in matrices. Nanoparticles embedded in a matrix are interesting from the experimental point of view since the position of the resonance can be tuned by changing the dielectric surrounding. They constitute a complicated system, where the plasmon lifetime strongly depends on the properties of the matrix, an effect that has been called chemical interface damping 1220 . The application of the TDLDA, within a simple model of the potential at the surface, confirms that the size-oscillation of the level-width predicted by our analytical method persists even for embedded clusters. 


\section{LANDAU DAMPING IN NANOPARTICLES}

The nanoparticles we consider are much smaller than the wavelength of the radiation, and the optical absorption cross-section at frequency $\omega$ is given by

$$
\sigma(\omega)=\frac{4 \pi e^{2} \omega}{3 c} \sum_{j}|\langle j|z| 0\rangle|^{2} \delta\left(E_{j}-\hbar \omega\right),
$$

where the dipole matrix element is taken between the electronic many-body ground state $|0\rangle$ and the excited states $|j\rangle$ with energy $E_{j}$ (the $z$-axis is chosen parallel to the electric field direction). The spectrum is dominated by the collective surface plasmon excitation which is characterized by an oscillation of the center of mass of the electrons with respect to the lattice of positive ions. Following Mie's classical theory 3 , for a nanoparticle in vacuum one expects the surface plasmon resonance at the frequency $\omega_{\mathrm{M}}=\omega_{\mathrm{p}} / \sqrt{3}$, where $\omega_{\mathrm{p}}$ is the bulk plasmon frequency of the material.

When the confinement is not parabolic the surface plasmon is not an eigenstate of the many-body Hamiltonian and its coupling to the particle-hole excitations leads to a broadening of the corresponding resonance in the spectrum (see the inset of Fig. 11).
A common approach to the plasmon lifetime consists in treating the collective excitation as an external perturbation which can give rise to the creation of electron-hole excitations $₫$. Within this framework, Fermi's Golden Rule yields the linewidth

$$
\Delta \Gamma(R)=2 \pi \sum_{p h}|\langle p|\delta V| h\rangle|^{2} \delta\left(\hbar \omega_{M}-\epsilon_{p}+\epsilon_{h}\right)
$$

where $|p\rangle$ and $|h\rangle$ are electron and hole states in the self-consistent field, respectively, and $\delta V$ is the dipole field due to the surface plasmon. More rigorous approaches, like discrete-matrix RPA would lead after certain approximations, to an equivalent separation of collective and particle-hole states.

In the case of spherical symmetry we can work in the effective one-dimensional problem of the radial coordinate for each value (in units of $\hbar$ ) of the angular momentum $L<\rho=R \sqrt{2 M E} / \hbar, E$ and $M$ being the energy and the effective mass of an electron, respectively, and $R$ denoting the radius of the nanoparticle. Assuming that the confinement and the interactions lead to hard walls at radius $R$ in the self-consistent field, and integrating over the electron-hole states, one obtains 9

$$
\Delta \Gamma(R)=C \gamma \int_{E_{\mathrm{F}}}^{E_{\mathrm{F}}+\hbar \omega_{\mathrm{M}}} \mathrm{d} E \sum_{L} \sum_{L^{\prime}=L \pm 1}(2 L+1)\left(2 L^{\prime}+1\right)<L, 0 ; 1,0 \mid L^{\prime}, 0>^{2} E\left(E-\hbar \omega_{\mathrm{M}}\right) d_{L}(E) d_{L^{\prime}}\left(E-\hbar \omega_{\mathrm{M}}\right)
$$

where $\left\langle L, 0 ; 1,0 \mid L^{\prime}, 0\right\rangle$ is a Clebsch-Gordan coefficient, $\gamma=\left(2 \pi \hbar^{3}\right) /\left(3 N M^{2} \omega_{\mathrm{M}} R^{4}\right), C=4 M R^{2} / \hbar^{2}$, and $d_{L}(E)$ is the one-dimensional density of states.

\section{SEMICLASSICAL THEORY}

Our approach consists in the evaluation of (3) by using the semiclassical expressione 21

$$
d_{L}(E)=\frac{\tau(E, L)}{2 \pi \hbar}\left(1+2 \sum_{r=1}^{\infty} \cos \left[r\left(\frac{S(E, L)}{\hbar}-\pi\right)\right]\right)
$$

which decomposes $d_{L}(E)$ in its smooth and oscillating components. Here, $\tau(E, L)=\hbar \sqrt{\rho^{2}-L^{2}} / E$ is the period of the one-dimensional motion,

$$
S(E, L)=2 \hbar\left[\sqrt{\rho^{2}-L^{2}}-L \arccos (L / \rho)\right]
$$

is the corresponding action, and the sum runs over all numbers of repetitions $r$. Integration over $L$, Poisson's summation rule, and a stationary phase apprgximation, lead to the semiclassical Berry-Tabor formula22, expressing the density of states of a sphere in terms of classical periodic trajectories. Taking only into account the smooth part of $d_{L}(E)$ in Eq. (3), we recover the wellknown $1 / R$ dependence

$$
\Gamma_{0}(R)=\frac{3 \hbar}{4} \frac{v_{\mathrm{F}}}{R} g(\xi)
$$

where $\xi=\hbar \omega_{\mathrm{M}} / E_{\mathrm{F}}, g(\xi)$ is a smoothly decreasing function with $g(0)=1$, and $v_{\mathrm{F}}\left(E_{\mathrm{F}}\right)$ is the Fermi velocity (energy).

Most interestingly, the oscillating part of the density of states gives rise to additional new features, namely an oscillation of $\Delta \Gamma(R)$ around $\Gamma_{0}(R)$ as a function of the radius. While the cross products between the smooth and the oscillating parts of $d_{L}(E)$ and $d_{L^{\prime}}\left(E-\hbar \omega_{\mathrm{M}}\right)$ can be neglected after the energy integration, the product of the two oscillatory components, after doing the $L$ summation with the aid of Poisson's summation rule, yields 


$$
\begin{aligned}
\Gamma_{\text {osc }}(R)= & \frac{2 \gamma}{\pi^{2}} \sum_{m=-\infty}^{\infty} \int_{\rho_{\min }}^{\rho_{\max }} \mathrm{d} \rho \rho \int_{-1 / 2}^{\rho} \mathrm{d} y \sum_{y^{\prime}=y \pm 1} f\left(y^{\prime}\right) \sqrt{\rho^{2}-y^{2}} \sqrt{\rho^{\prime 2}-y^{\prime 2}} \\
& \times \sum_{r=1}^{\infty} \sum_{r^{\prime}=-\infty}^{\infty}(-1)^{r+r^{\prime}} \cos \left[r S(\rho, y)+r^{\prime} S\left(\rho^{\prime}, y^{\prime}\right)+2 \pi m y\right]
\end{aligned}
$$

with $\rho^{\prime}=\sqrt{\rho^{2}-\left(k_{\mathrm{F}} R\right)^{2} \xi}$. The limits of the $\rho$-integral are given by $\rho_{\min }=\max \left(k_{\mathrm{F}} R, k_{\mathrm{F}} R \xi\right)$ and $\rho_{\max }=k_{\mathrm{F}} R \sqrt{1+\xi}$. We have defined $f\left(y^{\prime}\right)=y^{\prime}+1$ for $y^{\prime}=y-1$ and $f\left(y^{\prime}\right)=y^{\prime}$ in the case $y^{\prime}=y+1$ ( $k_{\mathrm{F}}$ is the Fermi wave-vector). The dominant terms correspond to $m=0, r=-r^{\prime}$ and $\bar{y}=\bar{y}^{\prime}+1=\rho /\left(\rho-\rho^{\prime}\right)$, yielding

$$
\Gamma_{\text {osc }}(R)=\frac{2 \gamma}{\pi^{3 / 2}} \int_{\rho_{\min }}^{\rho_{\max }} \mathrm{d} \rho \frac{\rho^{7 / 2} \rho^{\prime 3 / 2}\left(\left(\rho-\rho^{\prime}\right)^{2}-1\right)^{5 / 4}}{\left(\rho-\rho^{\prime}\right)^{4}} \sum_{r=1}^{\infty} \frac{1}{\sqrt{r}} \cos \left[2 r\left(\frac{\left(k_{\mathrm{F}} R\right)^{2} \xi-2 \bar{y}+1}{\sqrt{\rho^{2}-\bar{y}^{2}}+\sqrt{\rho^{\prime 2}-\bar{y}^{\prime 2}}}\right)\right] .
$$

Since $\rho$ is of the order of $k_{\mathrm{F}} R$, and $k_{\mathrm{F}} R \xi \gg 1$, the argument of the cosine is close to $2 r k_{\mathrm{F}} R \xi$, and (6) yields a contribution to $\Delta \Gamma$ which oscillates as a function of the radius of the nanoparticle. It is important to note that this oscillatory behavior is due to the angular-momentum restricted electron-hole density-density correlation, and therefore more subtle than the well-known oscillations of the density of states with the energy.

Fig. 11 shows the result for $\Delta \Gamma=\Gamma_{0}+\Gamma_{\text {osc }}$ which is obtained by numerically evaluating the $\rho$-integral in Eq. (6). The typical amplitude of the oscillations can be estimated by the value $\Gamma_{\mathrm{osc}}^{\max }=6 \sqrt{2 \pi} \hbar^{2} /\left(M R^{2} \sqrt{k_{\mathrm{F}} R \xi^{3}}\right)$, valid in the limit $k_{\mathrm{F}} R \xi^{2} \ll 1$. This shows that the oscillating term represents an important correction to $\Gamma_{0}(R)$ for small particle sizes.

Higher repetitions $(r>1)$ of the periodic orbits are suppressed in Eq. (6) because of the prefactor $1 / \sqrt{r}$, and the oscillations of the cosine as a function of $\rho$. Including a small intrinsic decoherence rate $\Gamma_{\mathrm{i}}$ accounts for the coupling to the environment and leads to an additional factor $\exp \left(-\Gamma_{\mathrm{i}} r R / h v_{\mathrm{F}}\right)^{23}$, which further reduces the oscillatory contributions from long trajectories with large repetition numbers $r$.

\section{NUMERICAL CALCULATIONS}

In order to study the relevance of the oscillatory behavior in more general situations, we performed quantum numerical calculations within a spherically symmet ric jellium model, using the linear response TDLDA 1.24 . The diagonalization of the Hamiltonian in the space of particle-hole excitations amounts to use the Random Phase Approximation in the self-consistent field with a local exchange potential. Further refinements of the pmerical approach (like the Self-Interaction Correction25) do not provide substantial changes in the effects we are interested in. Because of the spherical symmetry of the model, we considered nanoparticle sizes corresponding to magic numbers of atoms where our numerical approach

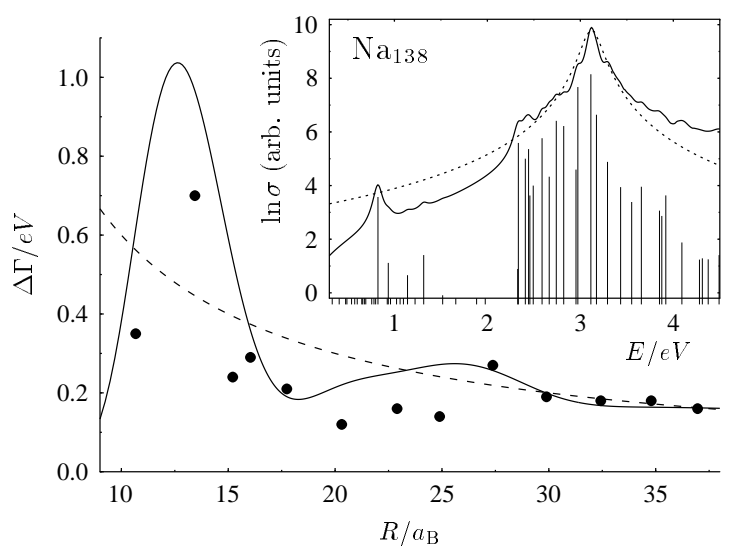

FIG. 1: Linewidth $\Delta \Gamma(R)$, as a function of the radius (in units of the Bohr radius $a_{\mathrm{B}}=0.53 \AA$ ), for Na nanoparticles containing between 20 and 832 atoms. The dots corresponding to TDLDA calculations are compared to the result of Eq. (6) (full line). The smooth term $\Gamma_{0}(R)$ is given by Eq. (四) (dashed). Inset: Logarithm of the numerically calculated absorption cross-section for $\mathrm{Na}_{138}$, showing a pronounced surface plasmon resonance, fitted by a Lorentzian (dotted line). The excited states are indicated by tick marks and their oscillation strengths are given by the height of the vertical lines.

is expected to work the best 22 .

\section{A. Alkaline metals}

A typical spectrum of the photo absorption crosssection (Eq. (11)) is shown in the inset of Fig. 1 for the example of a sodium cluster, where a non-zero $\Gamma_{\mathrm{i}}$ smears the singularities of the spectrum. The collective excitation can be identified as the main peak of the spectrum (note that the logarithm of $\sigma$ is plotted). The vertical lines are essentially given by single electron-hole excitations, except for the one at the center of the surface plasmon resonance, which is the superposition of many such ex- 


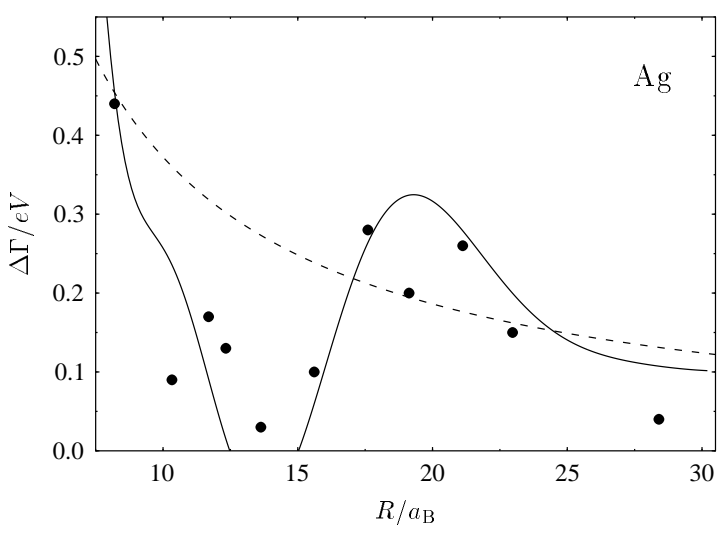

FIG. 2: The dots represent the numerically obtained surface plasmon linewidth for the case of silver nanoparticles embedded in an argon matrix, as a function of their radius $R$ in units of the Bohr radius. The dashed and the solid lines represent $\Gamma_{0}(R)$ and $\Delta \Gamma(R)$, respectively, reduced by a global factor of 3 with respect to Eqs. (4) and (6) (see text).

citations. The surface plasmon linewidth $\Gamma$, represented by thick dots in Fig. 11, is obtained from a Lorentzian fit to the main peak. We chose an intrinsic width $\Gamma_{\mathrm{i}}$ larger than the typical distance in the electron-hole spectrum and verified that $\Delta \Gamma(R)$ is approximately independent of the precise value of $\Gamma_{\mathrm{i}}$. Confirming our analytical calculations, at small $R$ the numerical results show strong deviations from $\Gamma_{0}(R)$ (dotted line) and a non-monotonic behavior as a function of the radius. Similar results are obtained for other alkaline metals. It should be mentioned that the phase of the semiclassical result from Eq. (6) is meaningful up to a constant shift which was adjusted according to the numerical data.

\section{B. Noble metals and embedded clusters}

Noble metals are more difficult to describe, due to band-structure effects and the fact that the comparison with experiments has to take into account the confinement of the particles in a matrix. In our numerical approach we included the contribution $\epsilon_{\mathrm{d}}$ of the d-electrons to the dielectric function of the metal particles, as well as the dielectric constant $\epsilon_{\mathrm{m}}$ of the matrix. For the resulting residual interaction we used a multipolar expansion as in Ref.27. We have checked that ipluding an interstitial spacing (the so-called 3- $\epsilon$ modeles) does not change significantly our results.

In Fig. 2 we show the numerical results (thick dots) for silver nanoparticles embedded in an inert matrix $\left(\mathrm{Ar}, \epsilon_{\mathrm{m}} \approx 1.75\right)$, exhibiting pronounced oscillations of $\Delta \Gamma(R)$ with the particle size, much like in the case of alkaline metals. The smooth part of the linewidth $\Gamma_{0}(R)$ (Eq. (4)), and our semiclassical result for $\Gamma_{\text {osc }}(R)$ (Eq. (6)) are an overall factor of 3 larger than the numer- ical results. This discrepancy is not surprising given the stringent approximations involved in both results, which are expected to be less applicable for embedded noble metal clusters, than for free alkaline metals. Nevertheless, the analytically calculated $\Gamma_{\text {osc }}(R)$ exhibits oscillations with the correct period, and its relative size (with respect to $\left.\Gamma_{0}(R)\right)$ agrees with the numerical results.

Reactive (non-inert) matrices like glass are often studied experimentally 2 , but still more difficult to analyze. By taking into account a high-energy conduction band in the insulaton 29, we extended our numerical calculations to this case 30 . These electronic states provide additional decay channels for the surface plasmon and its lifetime is considerably reduced. Our results for the Landau damping contribution to the linewidth of Ag nanoparticles embedded in $\mathrm{SiO}_{2}$ amount to about one half of the experimental linewidth, but still show strong size-dependent oscillations, as in the previous cases.

\section{CONCLUSIONS}

In conclusion, we have found a mechanism leading to an important oscillatory contribution to the Landau damping linewidth of the collective surface plasmon excitation in small metallic nanoparticles. The oscillations of the linewidth as a function of the particle size arise from the oscillations of the angular-momentum restricted electron-hole density-density correlations, that we calculate within a semiclassical approach. We obtain a quantitative agreement between analytical and numerical (TDLDA) results for free alkaline metallic clusters, and a qualitative agreement for more complicated cases.

A systematic experimental investigation of the linewidth oscillations extending the results for specific situations reported in Refs.13.14.15 would provide an important step towards the understanding of the rich physics involved in the collective excitations of finite systems. A small inhomogeneous broadening will not kill the effect if the size dispersion of the nanoparticles is smaller than the period of the oscillations (approximately $\left(k_{\mathrm{F}} \xi\right)^{-1}$, according to Eq. (6)). Moreover, recent experimental developments make it possible to study the homogeneous broadening of the murface plasmon also in electrically uncharged clusters 31 [22. In the case of clusters embedded in a matrix, the oscillations of the width of the plasmon can be seen not only as a function of the size but also as a function of the dielectric constant of the environment. Changing $\epsilon_{\mathrm{m}}$ shifts the position of the plasmon, without considerably modifying the single-particle part of the spectrum. Therefore the plasmon linewidth varies according to the position of the resonance with respect to the particle-hole excitation spectrum. 


\section{Acknowledgments}

We thank J.-Y. Bigot, M. Brack, J. Feldmann, L. Guidoni, H. Haberland, P.A. Hervieux, and D. Ullmo for helpful discussions. Financial support from the European Union through the TMR/RTN program is acknowledged.
${ }^{1}$ G.F. Bertsch and R.A. Broglia, Oscillations in Finite Quantum Systems, Cambridge University Press (Cambridge, 1994).

2 K. L. G. Heyde, The Nuclear Shell Model, Springer-Verlag (Heidelberg, 1994).

3 G. Mie, Ann. Phys. (Leipzig) 25, 377 (1908).

${ }^{4}$ U. Kreibig and M. Vollmer, Optical Properties of Metal Clusters, Springer (Berlin, 1995).

${ }^{5}$ C. Ellert, M. Schmidt, C. Schmitt, T. Reiners, and H. Haberland, Phys. Rev. Lett. 75, 1731 (1995); H. Haberland, in Metal Clusters, ed. by W. Ekardt, John Wiley (New York, 1999).

${ }^{6}$ A. Kawabata and R. Kubo, J. Phys. Soc. Jpn. 21, 1765 (1966).

7 W. Kohn, Phys. Rev. 123, 1242 (1964).

8 J.F. Dobson, Phys. Rev. Lett. 73, 2244 (1994).

9 C. Yannouleas, Nucl. Phys. A 439, 336 (1985); C. Yannouleas and R.A. Broglia, Ann. Phys. (N.Y.) 217, 105 (1992).

10 M. Barma and V. Subrahmanyam, J. Phys.: Condens. Matter 1, 7681 (1989).

11 C. Yannouleas, E. Vigezzi, and R.A. Broglia, Phys. Rev. B 47, 9849 (1993); C. Yannouleas, Phys. Rev. B 58, 6748 (1998).

12 U. Kreibig and L. Genzel, Surf. Sci. 156, 678 (1985).

13 K.P. Charlé, W. Schulze, and B. Winter, Z. Phys. D 12, 471 (1989).

14 C. Bréchignac, Ph. Cahuzac, J. Leygnier, and A. Sarfati, Phys. Rev. Lett. 70, 2036 (1993).

15 S. Mochizuki, M. Sasaki, and R. Ruppin, J. Phys.: Condens. Matter 9, 5801 (1997).
16 W. Kleinig, V.O. Nesterenko, P.-G. Reinhard, and Ll. Serra, Eur. Phys. J. D 4, 343 (1998).

17 J. Babst and P.-G. Reinhard, Z. Phys. D 42, 209 (1997).

18 W. Ekardt, Z. Phys. B 103, 305 (1997).

19 W. Ekardt, Phys. Rev. B 31, 6360 (1985).

${ }^{20}$ H. Hövel, S. Fritz, A. Hilger, U. Kreibig, and M. Vollmer, Phys. Rev. B 48, 18178 (1993).

21 M.C. Gutzwiller, Chaos in Classical and Quantum Mechanics (Springer-Verlag, Berlin, 1990).

22 M.V. Berry and M. Tabor, Proc. R. Soc. London, Ser. A, 349, 101 (1976).

23 J. Lermé, Ch. Bordas, M. Pellarin, B. Baguenard, J.L. Vialle, and M. Broyer, Phys. Rev. B 48, 9028 (1993).

${ }^{24}$ G. Bertsch, Comp. Phys. Comm. 60, 247 (1990).

25 M. Madjet, C. Guet, and W.R. Johnson, Phys. Rev. A 51, 1327 (1995).

${ }^{26}$ Incompletely filled shells fesult in a non-spherical shape and a split resonance peak 5 . This obvious shell effect adds up to the oscillations that we have discussed.

27 A. Rubio and L. Serra, Phys. Rev. B 48, 18222 (1993).

28 J. Lermé, B. Palpant, B. Prével, E. Cottancin, M. Pellarin, M. Treilleux, J.L. Vialle, A. Perez, and M. Broyer, Eur. Phys. J. D 4, 95 (1998).

29 B.N.J. Persson, Surf. Sci. 281, 153 (1993).

30 R.A. Molina, D. Weinmann, and R.A. Jalabert, unpublished.

31 T. Klar, M. Perner, S. Grosse, G. von Plessen, W. Spirkl, and J. Feldmann, Phys. Rev. Lett. 80, 4249 (1998).

32 F. Stietz, J. Bosbach, T. Wenzel, T. Vartanyan, A. Goldmann, and F. Träger, Phys. Rev. Lett. 84, 5644 (2000). 